\title{
ESTIMATION OF SALIVARY LEVELS OF INTERLEUKINE-6 (IL-6) AND INTERLEUKINE-8 (IL-8) IN THE ORAL PRE-MALIGNANT AND MALIGNANT LESIONS
}

\author{
Abdel-Fattah M.*, El Gaaly K. ${ }^{* *}$, Al-Refai M. ${ }^{* * *}$ and Galwash D ${ }^{* * * *}$
}

\begin{abstract}
The present investigation was designed to evaluate salivary IL-8 and IL-6 levels in premalignant and OSCC patients, in order to verify the effectiveness of using them as diagnostic biomarkers for detection of OSCC and possibly early malignant transformation in premalignant lesions. This study utilized ELISA technique in order to assess the levels of IL-8 and IL-6 in whole unstimulated saliva. The ELISA system is cited as the most sensitive, well-established, and widely available protein-based testing platform for the detection of specific proteins in body fluids or tissue. The present study was carried out on 50 subjects where they were subdivided into 15 patients suffering from frank oral malignancy, 20 patients with oral premalignant lesions. Among the 20 patients, biopsy results enabled us to further subdivide the group into patients with dysplastic lesions and others with no dysplastic changes; 10 patients into each group. And 15 systemically healthy control. The control group was selected to match the premalignant and malignant groups regarding age and gender, with no oral mucosal lesions. The results showed that salivary IL-6 and IL-8 levels were higher in all the studied groups. Also the control group had the lowest level of IL-6 and IL-8 in the saliva. As for the OSCC group they showed the highest levels of IL-6 and IL- 8 in the saliva when compared to both the OPL and the control groups, thus it could be concluded that salivary IL-6 and IL- 8 might be a potential biomarker for the detection of OSCC. So, salivary IL-6 and IL-8 hold promise as biomarkers for OSCC. And also, analysis of cytokines as IL-6 and IL-8 in saliva may be one of predictive means to reflect the trend of malignant transformation of oral premalignant lesions
\end{abstract}

* Assistant lecturer, Oral Medicine, Oral Diagnosis and Periodontology Department, Faculty of Dentistry, MSA University, $6^{\text {th }}$ October, Egypt.

** Professor of Oral Medicine, Oral Diagnosis and Periodontology, Faculty of Oral and Dental Medicine, Cairo University, Egypt.

*** Professor of Oral Medicine, Oral Diagnosis and Periodontology Faculty of Oral and Dental Medicine Cairo University, Egypt.

**** Ass. Prof. Oral Medicine and Periodontology. MSA University . 


\section{INTRODUCTION}

Oral cancer refers to all malignancies arising from the lips and the oral cavity. It affects more than 481,000 new patients worldwide. It is the sixth most common cancer in the USA The $90 \%$ of oral cancers are oral squamous cell carcinoma. This cancer, when found early, has an 80 to $90 \%$ survival rate. Despite this fact and the great treatment advances, the World Health Organization has reported oral cancer as having one of the highest mortality ratios amongst other malignancies with a death rate at five years from diagnosis at $45 \%$. This high morbidity rate can definitely be attributed to the late diagnosis of the disease (Boyle P et al., 2008).

Saliva has been long proposed and used as a diagnostic medium because it is easily accessible and its collection is non-invasive, not time-consuming, and inexpensive, requires minimal training and can be used for the mass screening of large population samples (Hu et al., 2007).

Saliva has gained notable attention as a diagnostic fluid because of its simple collection and processing, minimal invasiveness and low costs. Many researchers have studied salivary proteins as potential diagnostic markers for various diseases such as breast cancer, ovarian cancer, Sjögrens syndrome, hepatocellular carcinoma, leukoplakia and oral cancer these potential disease markers, if successfully developed, can lead to simple clinical tools for early detection and the monitoring of disease prognosis and treatment in saliva (Hu et al., 2007).

Using salivary biomarkers for oral squamous cell carcinoma (OSCC) detection is the facilitation of diagnostics at a point where OSCC is still small or in oral premalignant lesions (OPML) so the treatment is very likely to be successful. In order to achieve this highly desirable goal, a saliva screening method must have sufficient sensitivity and specificity, be rather inexpensive, noninvasive, have high-throughput, and can be used by non-trained personnel. Using the latest advancements in technology could help put saliva in such a clinical context. Several salivary protein markers for OSCC and oral premalignant lesions (OPML) have been investigated in various studies and have shown relatively moderate sensitivity and specificity values (Hu et al., 2007).

It has been proven that NF-kappa B-dependent cytokines interleukins (IL-1, -6, -8, and tumor necrosis factor (TNF)- $\alpha$ ) are produced by OSCC cells. Furthermore, expression profiling of OSCC using microarray indicated that IL-6 and -8 are significantly over expressed by these tumors (Malamud D,1992).

Martin D et al. (2009) investigated whether interleukin (IL)-6 and/or IL-8 could serve as informative biomarkers for OSCC in saliva. Interleukin 8 was detected at higher concentrations in saliva, while IL-6 was detected at higher concentrations in serum of patients with OSCC. Thus, they concluded that IL-8 in saliva and IL-6 in serum hold promise as biomarkers for OSCC.

\section{SUBJECTS AND METHODS}

The present study was performed on a total of 50 individuals selected from the out-patient clinic of Oral Medicine and Periodontology Department, Faculty of Oral and Dental Medicine and from the Surgical Oncology Department in the National Cancer Institute, Cairo University. All subjects included in this study were systemically free according to the modified Cornell's Medical index (Kerr and Millard, 1965).

\section{The subjects were subdivided into three groups}

Group I: Included 15 patients suffering from oral squamous cell carcinoma.

Group II: included 20 patients suffering from OPL lesions. Among the 20 patients, biopsy results enabled us to further subdivide the group into patients with dysplastic lesions and others 
with no dysplastic changes; 10 patients into each group.

Group III: included 15 individuals age, gender and periodontal status matched with OSCC and OPL patients and not suffering from any oral mucosal lesions. These acted as control group. All the patients and controls were subjected to the following:

- Case history: Comprehensive oral diagnosis was carried out for all participating individuals using the Department of Oral Diagnosis chart.

- Clinical examination: The oral cavities of all individuals were carefully examined. Group I (OSCC patients) and group II (OPL patients) included 20 cases with premalignant lesions as lichen planus, actinic keratosis, leukoplakia and erythroplakia. Control subjects were examined carefully to confirm the absence of any mucosal lesion and to assess their periodontal status.

- Biopsy: specimens were obtained from all OSCC and OPL lesions to confirm the diagnosis and the presence of dysplastic changes that might indicate early malignant transformation in the premalignant lesions (after patients' consent). For biopsies to be taken from the lesions, ring block anesthesia was performed. There was no direct infiltration of the anesthetic solution into the examined areas to avoid its direct damage. A surgical double wedge incisional biopsy was carried out to a depth of about $2 \mathrm{~mm}$ (Colema et al., 1999).

- Histopathologic evaluation: Prepared sections were examined in order to confirm the clinical diagnosis. Biopsy specimens were immediately fixed in $10 \%$ neutral buffered formalin for 48 hrs and then processed in the routine way for preparing a paraffin block. Five microns thick sections were cut and stained with conventional Hematoxylin \& Eosin (H\&E) for histopathologic examination.
- Salivary sample collection: Collection of whole unstimulated saliva (WUS) using standard techniques was done as described by Navazesh (1993). Briefly, subjects refrained from eating, drinking, using chewing gum etc., for at least $1 / 2$ $\mathrm{h}$ prior to the evaluation. Samples were obtained by requesting subjects to swallow first, tilt their head forward and expectorate all saliva in a tube for 5 minutes without swallowing. After collection, all samples were immediately stored at $-20 \mathrm{C}$ until assayed.

- Detection of salivary IL-6 and IL-8 in all samples: Levels of IL-6 and IL-8 were measured in all salivary samples by using an ELISA kit (Orgenium laboratories, Business Unit, Finland). The principles of the test involve a sandwich-type ELISA where a polyclonal anti-antibody, adsorbed onto microwells, binds IL-6 and IL-8 in the samples. Salivary samples were centrifuged for $2 \mathrm{~min}$.at $10.000 \mathrm{xg}$ and the clarified supernatant was filtered through a 0.45 $\mu \mathrm{m}$ low protein binding membrane, separated into $0.5 \mathrm{ml}$ aliquots and frozen at $-80^{\circ} \mathrm{C}$ until use for quantitation of salivary levels of IL- 6 and IL- 8 by ELISA.

Calculation of results: The average absorbance values were calculated for each set of duplicate standards, samples and controls. Duplicates should be within 20 percent of the mean .A standard curve was plotted; the mean absorbance for each standard on the Y-axis against the concentration on the $\mathrm{X}$-axis and a best fit curve was drawn through the points on the graph. The unknown concentrations of the samples were calculated from the standard curve. To determine the concentration of IL- 8 and IL-6 in each sample, first the mean OD value was found on the ordinate and a horizontal line was extended to the standard curve. At the point of intersection, a vertical line was extended to the abscissa and the corresponding Il-8 and IL-6 concentrations were read. 


\section{RESULTS}

\section{Demographic data}

There was no statistically significant difference between mean age values in the four groups. There was also no statistically significant difference between gender distributions in the four groups. Descriptive statistics and results of comparison between demographic data in the four groups. The mean and standard deviation for all groups and as seen, the highest value was detected in the oral carcinoma group which was $47.7 \pm 14.1$, the lowest mean was detected by control group $39.7 \pm 14.8$, where the pre malignant lesions group registered values $44.8 \pm 10.8$ for the OLP with dysplasia changes and $41.9 \pm 10.7$ foe OLP without dysplastic changes group (figure 1).

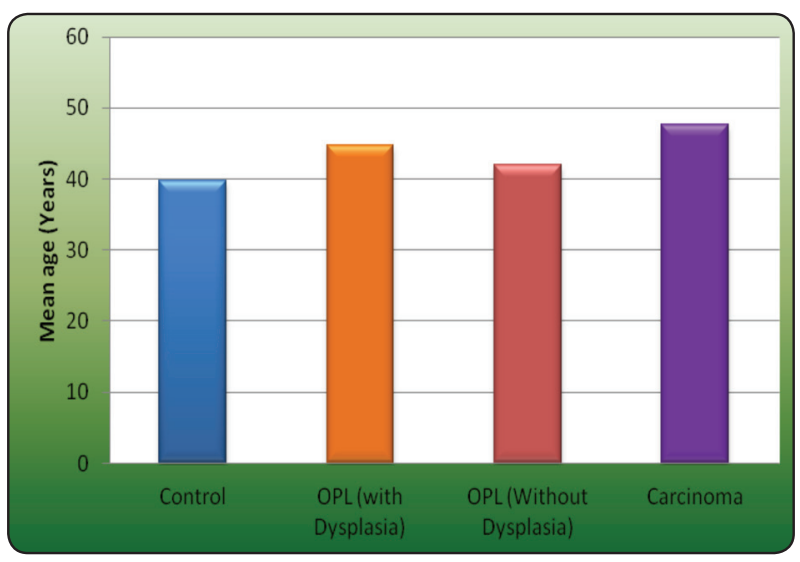

Fig. (1) Bar chart representing the mean of age values in the four groups

The gender distribution among the patients group where 9 females (60\%) and 6 males (40\%) in the oral malignant lesions group, 7 females $(70 \%)$ and 3 males $(30 \%)$ in the OLP without dysplasia changes group, 4 females (40\%) and 6 males (60\%) in the OLP with dysplastic changes group and 9 females $(60 \%)$ and 6 males $(40 \%)$ in the control group (figure 2).

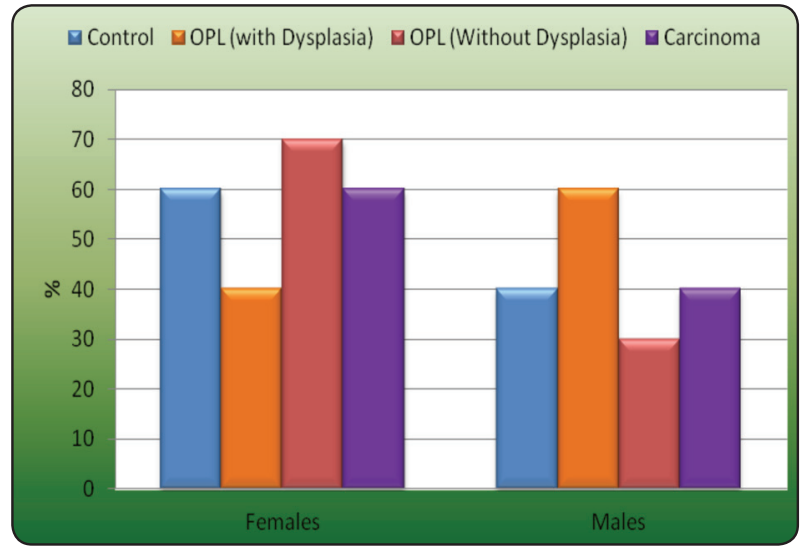

Fig. (2) Bar chart representing gender distributions in the four groups

\section{IL-8}

Statistical analysis of salivary IL-8 level in $\mathrm{pg} / \mathrm{ml}$ in the all groups. Carcinoma showed the statistically significantly highest mean IL-8 level 3244.1 (2967.6- 3376.0) pg/ ml with mean 3202.1 $\mathrm{pg} . / \mathrm{ml}( \pm 128.6)$. In the OLP without dysplasia the range was 910.0 (707.0-1077.3) $\mathrm{pg} / \mathrm{ml}$ with mean $903.6 \mathrm{pg} / \mathrm{ml}( \pm 130.6)$. The OLP with dysplasia group salivary IL-8 was range 2198.6 (1091.3$3078.7) \mathrm{pg} . / \mathrm{ml}$ dysplasia with mean $1950.2 \mathrm{pg} / \mathrm{ml}$ $( \pm 659.5)$, on the other hand, the control group the range was 328.4 ( 223.8-448.2) $\mathrm{pg} / \mathrm{ml}$ with mean range $327.7 \mathrm{pg} / \mathrm{ml}( \pm 327.7)$.carcinoma showed the statistically significantly highest mean of salivary level of IL-8. OLP with dysplasia showed statically significantly lower mean value followed by OLP without dysplasia, control group showed lowest level (p value <0.001) (figure 3).

\section{IL-6}

Statistical analysis of salivary IL-6 level in $\mathrm{pg} / \mathrm{ml}$ in the all groups. Carcinoma showed the statistically significantly highest IL-6 level 73.2 (52.4-101.2) $\mathrm{pg} / \mathrm{ml}$ with mean $72.0( \pm 13.8) \mathrm{pg}$ $/ \mathrm{ml}( \pm 128.6)$. In the OLP without dysplasia the range was $23.3(20.3-25.3) \mathrm{pg} / \mathrm{ml}$ with mean $22.3( \pm 1.4) \mathrm{pg} / \mathrm{ml}$. The OLP with dysplasia group salivary IL-6 was range $39.4(32.4-55.4) \mathrm{pg} / \mathrm{ml}$ 
with mean $40.5( \pm 8.0) \mathrm{pg} / \mathrm{ml}$, on the other hand the control group the range was $5.7(1.4-15.6) \mathrm{pg} . /$ $\mathrm{ml}$ with mean range $6.7( \pm 3.9) \mathrm{pg} / \mathrm{ml}$. Carcinoma showed the statistically significantly highest mean IL-6 level. OPL with dysplasia showed statistically significantly lower mean value followed by OPL without dysplasia. Control group showed the statistically significantly lowest mean IL-6 level. (P value <0.001). (figure 4).

\section{Correlation between IL-8 and IL-6}

There was a statistically significant positive (direct) correlation between salivary IL-8 and IL-6. An increase in IL-8 levels is associated with an increase in IL-6 levels and vice versa. The correlation 0.923 and $(\mathrm{P}$-value $<0.001)$.

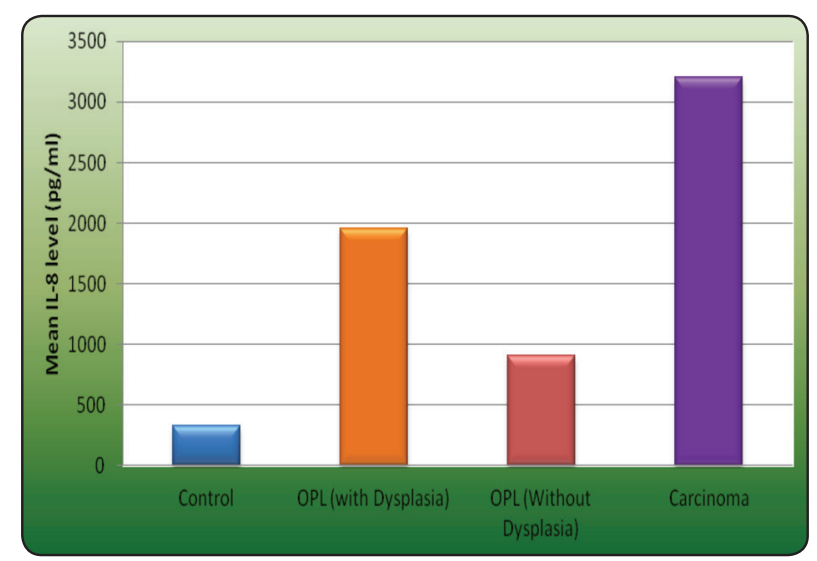

Fig. (3) Bar chart representing mean IL-8 levels in the four included groups

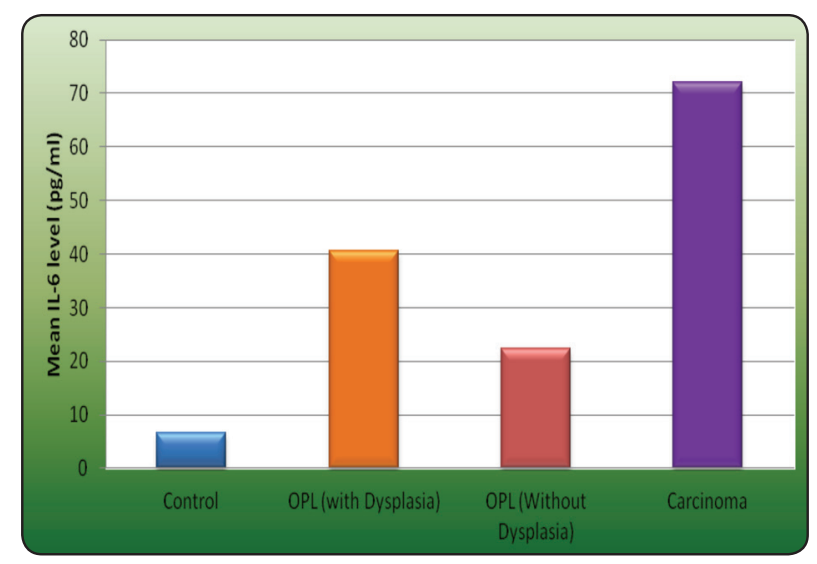

Fig. (4) Bar chart representing mean IL-6 levels in the four included groups.

\section{DISCUSSION}

Oral squamous cell carcinoma (OSCC) accounts for more than $94 \%$ of oral cavity and oropharyngeal cancer cases worldwide. Despite improvement in diagnosis and local disease management, longterm survival rates in patients with OSCC have not increased significantly over the last 30 years and are among the lowest of the major cancers mainly due to the fact that most patients are diagnosed at advanced disease stages (Duffy et al., 2008).

This diagnostic delay may be caused by either the patients (who may not report unusual oral features) or by health care workers (who may not investigate observed lesions thoroughly). It is presumed that such delays are longer for asymptomatic lesions (Mehrotra et al., 2006; Messadi et al., 2009).

A significant proportion of OSCCs develop from premalignant lesions such as leukoplakia, lichen planus and oral submucous fibrosis (Mehrotra et al., 2006). Although rather easily accessible compared to other cancers, the diagnosis of OSCC can be challenging since most lesions are small and asymptomatic and are easily overlooked or misjudged. Early detection would have a great impact on survival, mortality and morbidity of OSCC (Brinkmann et al., 2011).

Dentists can increase survival rates, and decrease the morbidity associated with the treatment of oral cancer, if lesions are detected at an early stage, or preferably if the precursor lesion is discovered, diagnosed, treated and monitored for malignant progression. For those where malignancy is detected early, soon after transitioning from pre-malignancy, treatment is more effective, and consequently the survival rate increases to about $80 \%$ (McCullough et al., 2010).

Early detection of cancer would also lead to fewer side effects from cancer treatments such as chemotherapy and radiotherapy and to a better prognosis. Moreover, oral cancer has a very 
high recurrence rate. Patients who survive a first encounter with this disease have up to a 20 folded increased risk of developing a second (recurrent) cancer (Wong, 2006; Seoane-Leston and DizDios, 2010).

One way of increasing the range of diagnostic options in the case of primary oral tumors and recurrence is to monitor the level of circulating tumor markers which have adequate sensitivity and specificity (Shah et al., 2011).

Ideally, biomarkers would provide information for the risk of transformation of a lesion in an individual, as well as correlate with severity of disease, prognosis, and response to treatment of cancer did arise (Carmeliet and Jain, 2011).

In addition, because biomarkers are products of malignant cells, they may represent a target for intervention for prevention of disease or for therapy and contribute to an understanding of the molecular mechanisms of the disease (Carmeliet and Jain, 2011; Pohl et al., 2011).

Saliva has an emerging role for the investigation of such circulating biomarkers which have relatively better sensitivity and specificity with regards to diagnosis, prognosis and treatment monitoring of the disease (Shah et al., 2011).

IL-6 stimulates tumor cell proliferation IL-6 is an important regulator of cell survival. Additionally, the physiological role of IL-6 has been shown to promote not only tumors proliferation, but also metastasis and symptoms of cachexia (Smith et al., 2001; Ara et al., 2009).

IL-6 is involved in both prevention and promotion of lymphomagenesis (Gilbert, L.A. and Hemann, M.T. 2012). IL-6 also plays a role in promoting tumorigenesis in tissues such as liver and colon (Jan Mauer et al., 2015).

On the other hand, IL-8 is associated with the promotion of neutrophil chemotaxis and degranulation and activation of multiple intracellular signaling pathways. Increased expression of IL-8 and/or its receptors has been characterized in cancer cells and tumor-associated macrophages, suggesting that IL-8 may function as a significant regulatory factor within the tumor microenvironment (Waugh and Wilson, 2008).

In addition, IL- 8 has been reported to contribute to tumor-derived angiogenic activity in a variety of human tumors (Veltri et al., 1999; Uehara et al., 2005; Rao et al., 2010).

The results of the present study showed that in all the included groups the IL-8 levels in saliva were higher in OSCC group when compared to both OPL and control groups. Where OSCC group showed the statistically significantly highest mean IL-8 level. OPL with dysplasia showed statistically significantly lower mean value than OSCC group followed by OPL without dysplasia. While the control group showed the statistically significantly lowest mean IL-8 level of all groups.

These results were in accordance with a previous study conducted by Punyani and Sathawane, (2013) as they reported a statistically significant increase in salivary IL-8 levels in OSCC group when compared to precancer group and control. They also reported that this increase in salivary IL-8 levels was directly proportional to the TNM staging of OSCC patients.

On the other hand, regarding IL-6 levels OSCC group showed the statistically significantly highest mean IL-6 level. OPL with dysplasia showed statistically significantly lower mean value than OSCC group followed by OPL without dysplasia. Control group also showed the statistically significantly lowest mean IL-6 level of all groups, and this was in accordance with (Brailo et al., 2012) who evaluated salivary and serum IL6 levels among other cytokines in patients with leukoplakia and oral cancer and found that IL6 levels was significantly higher in oral cancer 
patients compared to patients with leukoplakia and control group.

In the present study there was a statistically significant positive (direct) correlation between IL-8 and IL-6. An increase in IL-8 levels is associated with an increase in IL-6 levels and vice versa.

The results of the present study were also in accordance with Brailo et al. (2012) who reported statistically significant increase in salivary IL-6 concentration in OSCC group when compared to control but the reported that the increase in IL-8 concentration was less significant. Also in line with our results John MA et al. (2004) studied salivary IL-8 and serum IL-6 concentrations in OSCC patients and concluded that IL-8 in saliva and IL-6 in serum hold promise as biomarkers for OSCC.

Al Talabani et al. (2009) also reported highly significant increase in levels of serum IL-6 and IL-8 detected in patients with OSCC in comparison to that of control group. On the other hand, Czerninski et al. (2013) reported higher serum levels for the cytokine biomarkers including IL6 , IL-8, but failed to discover statistically significant differences between normal, PMD (dysplasia or OLP), OSCC.

Another study conducted by Zhang et al. (2007) came out with similar findings to this study regarding IL-6 and IL-8 levels in premalignant lesions as it revealed that both salivary and serum levels of IL-6 and IL-8 were significantly elevated in the OLP patients as compared with those in the control group.

On the other hand, Cohen et al. (2009) studied the effect of curcumin as a new adjunctive treatment modality for HNSCC on IL-6 and IL-8 levels and showed a dose-dependent inhibition of IL-6 and IL-8 production with increasing concentrations of curcumin.

Moreover Rhodus et al. (2006) reported a significant decrease in pro-inflammatory cytokines as (TNF- $\alpha$, IL-1-a, IL-6 and IL-8) in OLP patients following treatment with topical dexamethasone mouthwash for 6 weeks. Accordingly IL-6 and IL-8 have the potential to be used as biomarkers to measure the efficacy of various treatment protocols.

In an attempt to determine a preliminary cut off point of IL-6 and IL-8 for diagnosis of OPL and OSCC in the present study a ROC Curve was created to estimate salivary IL-6 and IL-8 with the highest sensitivity and specificity which was $100 \%$ and $100 \%$ respectively. The most probable cut off point was estimated to be $15.6 \mathrm{pg} / \mathrm{ml}$ and $448.2 \mathrm{pg} / \mathrm{ml}$, respectively.

Collectively, our results, together with other studies, indicate that analysis of cytokines as IL-6 and IL-8 in saliva may be one of predictive means to reflect the trend of malignant transformation of oral premalignant lesions and they might have diagnostic and/or prognostic utility as surrogate indicators of carcinogenic transformation from oral premalignant lesions.

\section{CONCLUSIONS}

- Analysis of cytokines as IL-6 and IL-8 in saliva may be one of predictive means to reflect the trend of malignant transformation of oral premalignant lesions

- Detection of salivary IL-6 levels reaching the cut-off value of $15.6 \mathrm{pg} / \mathrm{ml}$ could indicate potential malignant transformation.

- Detection of salivary IL-8 levels reaching the cut-off value of $448.2 \mathrm{pg} . / \mathrm{ml}$ could indicate potential malignant transformation.

- Salivary IL-6 and IL-8 hold promise as biomarkers for OSCC.

- IL-6 and IL-8 have the potential to be used as biomarkers to measure the efficacy of various treatment protocols. 


\section{REFERENCES}

- Ara, T., Song, L., Shimada, H., Keshelava, N., Russell, H. V., Metelitsa, L. S., Groshen, S. G., Seeger, R. C. and DeClerck, Y. A. Interleukin-6 in the bone marrow microenvironment promotes the growth and survival of neuro blastoma cells. Cancer Res. 2009; 69: 329-337.

- Al Talabani , Shanaz M., Abdul-Wahab R. Serum and salivary levels of proinflammatory cytokines as potential biomarkers in the diagnosis of oral squamous cell carcinoma .J Bagh College Dentistry. 2009; 21(1): 60-65.

- Boyle JO, Mao L, Brennan JA, Koch WM, Eisele DW, Saunders JR, Sidransky D. Gene mutations in saliva as molecular markers for head and neck squamous cell carcinomas. Am. J. Surg. 1994; 168(5): 429-432.

- Brailo V, Boras VV, Arambasin AC, Alajbeg IZ, Milenovic A, Lukac J. The significance of salivary interleukin 6 and tumor necrosis factor alpha in patients with oral leukoplakia. Oral Oncol. 2012;42:370-373.

- Brinkmann O, Kastratovic DA, Dimitrijevic MV, Konstantinovic VS, Jelovac DB, Antic J, Nesic VS, Markovic SZ, Martinovic ZR, Akin D, Spielmann N, Zhou H, Wong DT. Oral squamous cell carcinoma detection by salivary biomarkers in a Serbian population. Oral Oncol 2011; 47(1): 51-55.

- Carmeliet P, Jain RK. Molecular mechanisms and clinical applications of angiogenesis. Nature 2011; 473: 298-307.

- Colema DV, Evans DM, Baker R (Eds). Histological methods and type of biopsy. Biopsy pathology and cytology of the cervix, 2nd edition. Arnold Company, London, pp. 40, 1999.

- Czerninski1 R, Basile2 JR, Kartin-Gabay1 T, Laviv3 A, Barak4 V. Cytokines and tumor markers in potentially malignant disorders and oral squamous cell carcinoma: a pilot study. Oral Diseases (2013) doi:10.1111/odi.12160.

- Duffy SA, Taylor JM, Terrell JE. Interleukin-6 predicts recurrence and survival among head and neck cancer patients. Cancer 2008; 113: 750-757.

- $\quad$ Gilbert, L.A. and Hemann, M.T. Context-specific roles for paracrine IL-6 in lymphomagenesis. Genes Dev. 2012; 26, 1758-1768

- Giannopoulou C, Kamma JJ, Mombelli A. Effect of inflammation, smoking and stress on gingival crevicular fluid cytokine level. J Clin Periodontol 2003; 30: 145-153.
- Hu S, Wang J, Meijer J, Ieong S, Xie Y, Yu T. Salivary proteomic and genomic biomarkers for primary Sjogren's syndrome. Arthritis Rheum. 2007; 56: 3588-3600.

- Jan Mauer, Jesse L. Denson, and Jens C. Brüning. Versatile functions for IL-6 in metabolism and cancer, Trends in Immunology February. 2015, Vol. 36, No. 2.

- Kerr DA, Millard HD. Oral Diagnosis 2nd edition. CV. Mosby Company, Saint-Louis, pp. 17, 1965.

- Martin D, Galisteo R, Gutkind JS. CXCL8/IL8 Stimulates Vascular Endothelial Growth Factor (VEGF) Expression and the Autocrine Activation of VEGFR2 in Endothelial Cells by Activating NFxB through the CBM (Carma3/ Bcl10/Malt1) Complex. J Biol Chem 2009; 284(10): 6038-6042.

- Malamud D. Saliva as a diagnostic fluid. Br Med J 1992; 305: 207-208.

- McCullough MJ, Prasad G, Farah CS. Oral mucosal malignancy and potentially malignant lesions: an update on the epidemiology, risk factors, diagnosis and management. Aust. Dent. J. 2010; 55 (1 Suppl): 61-65.

- Mehrotra R, Gupta A, Singh M, Ibrahim R. Application of cytology and molecular biology in diagnosing premalignant or malignant oral lesions. Mol Cancer. 2006; 5: 11.

- Mehrotra R. The role of cytology in oral lesions: a review of recent improvements. Diagn. Cyto. Pathol. 2012; 40: 73-83.

- Messadi DV, Wilder SP, Wolinsky L. Improving oral cancer survival: the role of dental providers. J Calif Dent Assoc 2009; 37: 789-98.

- Navazesh M. Methods for collecting saliva. Ann NY Acad Sci 1993; 694: 72-77.

- Pohl M, Werner N, Munding J. Tannapfel A, Graeven U, Nickenig G, Schmiegel W, Reinacher-Schick A. Biomarkers of anti-angiogenic therapy in metastatic colorectal cancer (mCRC): original data and review of the literature. Z Gastroenterol. 2011; 49: 1398-1406.

- $\quad$ Punyani SR, Sathawane RS. Salivary level of interleukin-8 in oral precancer and oral squamous cell carcinoma. Clin Oral Invest 2013; 17: 517-524.

- $\quad$ Rao SK, Pavicevic Z, Du Z, Kim JG, Fan M, Jiao Y, Rosebush M, Samant S, Gu W, Pfeffer LM and Nosrat CA. Pro-inflammatory Genes as Biomarkers and Therapeutic Targets in Oral Squamous Cell Carcinoma. J Biol Chem 2010; 285(42): 32512-32521. 
- $\quad$ Rhodus NL, Cheng B, Myers S, Miller L, Ho V, Ondrey F. The feasibility of monitoring NF-KB associated cytokines TNF-a, IL-1a, IL-6, and IL-8 in whole saliva for the malignant transformation of oral lichen planus. Mol Carcinog 2005; 44: 77-82.

- $\quad$ Rhodus NL, Ho V, Miller CS, Myers S, Ondrey F. NF-kB dependent cytokine levels in saliva of patients with oral preneoplastic lesions and oral squamous cell carcinoma. Cancer Detect Prev 2006; 29(1): 42-45.

- Seoane-Lestón J, Diz-Dios P. Diagnostic clinical aids in oral cancer. Oral Oncol. 2010; 46: 418-422.

- Shah FD, Begum R, Vajaria BN, Patel KR, Patel JB, Shukla SN, Patel PS. A Review on Salivary Genomics and Proteomics Biomarkers in Oral Cancer. Int. J. Clin. Biochem. 2011; 26(4): 326-334.

- $\quad$ Smith DR, Polverini PJ, Kunkel SL, Orringer MB, Whyte RI, Burdick MD, Wilke CA, Strieter RM. Inhibition of Interleukin 8 Attenuates Angiogenesis in Bronchogenic Carcinoma. J Exp Med 2001; 179: 1409-1415.

- St John MA, Li Y, Zhou X, Denny P, Ho CM, Montemagno C, Shi W, Qi F, Wu B, Sinha U, Jordan R, Wolinsky L, Park NH, Liu H, Abemayor E, Wong DT. Interleukin 6 and interleukin 8 as potential biomarkers for oral cavity and oropharyngeal squamous cell carcinoma. Arch Otolaryngol Head Neck Surg 2004; 130(8): 929-935.

- Uehara H, Troncoso P, Johnston D, Bucana CD, Dinney C, Dong Z, Fidler IJ, Pettaway CA. Expression of interleukin-8 gene in radical prostatectomy specimens is associated with advanced pathologic stage. Prostate 2005; 64: 40-49.

- Veltri RW, Miller MC, Zhao G, Ng A, Marley GM, Wright GL Jr, Vessella RL, Ralph D. Interleukin-8 serum levels in patients with benign prostatic hyperplasia and prostate cancer. Urology. 1999; 53: 139-147.

- Waugh DJ, Wilson C. The Interleukin-8 Pathway in Cancer. Clin. Cancer Res. 2008; 14(21): 6735-41.

- Wong DT. Towards a simple, saliva-based test for the detection of oral cancer. 'oral fluid (saliva), which is the mirror of the body, is a perfect medium to be explored for health and disease surveillance'. Expert Rev. Mol. Diagn. 2006a; 6: 267-272.

- Zhang Y, Lin M, Zhang S, Wang Z, Jiang L, Shen J, Bai J, Gao F, Zhou M, Chen Q. NF-KB-dependent cytokines in saliva and serum from patients with oral lichen planus: A study in an ethnic Chinese population. Cytokine .2007; 41: 144-149. 